\title{
Mesoporous Magnetic Gold "Nanoclusters" as Theranostic Carrier for Chemo-Photothermal Co-therapy of Breast Cancer
}

\author{
JinRong Peng1 ${ }^{*}$, TingTing $\mathrm{Qi}^{1^{*}}$, JinFeng Liao ${ }^{*}$, BingYang Chu' ${ }^{1}$, Qian Yang1, Ying $\mathrm{Qu}^{1}$, WenTing $\mathrm{Li}^{1}, \mathrm{He} \mathrm{Li}^{2}$, \\ Feng Luo ${ }^{1}$, ZhiYong Qian ${ }^{1}$ \\ 1. State Key Laboratory of Biotherapy, West China Hospital, West China Medical School, Sichuan University, Chengdu 610041, PR China; \\ 2. School of Chemistry and Chemical Engineering, University of Jinan, Jinan 250022, PR China. \\ * These authors have done equal contribution to this work.
}

$\triangle$ Corresponding author: Tel.: +86 28851640 63; fax: +86 28851640 60. E-mail: anderson-qian@163.com, zhiyongqian@scu.edu.cn (Z. Qian).

() Ivyspring International Publisher. This is an open-access article distributed under the terms of the Creative Commons License (http://creativecommons.org/ licenses/by-nc-nd/3.0/). Reproduction is permitted for personal, noncommercial use, provided that the article is in whole, unmodified, and properly cited.

Received: 2013.10.1I; Accepted: 2014.02.28; Published: 2014.04.04

\begin{abstract}
Photothermal therapy (PTT) is proved to be an efficient manner for superficial tumor therapy in preclinical studying. The tumor suppression of chemotherapy can be enhanced by combining with PTT. In this study, we reported a mesoporous magnetic gold "nanoclusters" (MMGNCs) structure as theranostic carrier for chemo-photothermal co-therapy. MMGNCs were successfully prepared and they exhibited efficient photo-thermal effect for PTT. The mesoporous structure provided MMGNCs with high drug loading capacity. By in vitro cytotoxicity testing, we revealed that the combination of PTT and chemotherapy could cause more damage than chemotherapy or PTT did alone. By topically targeting mediated by the extra-magnetic field (MF), MMGNCs can be targeted to the tumor site efficiently. In vivo chemo-photothermal co-therapy of 4TI breast cancer, under the combinational treatments of chemo-photothermal co-therapy and extra-MF targeting, the tumor growth has been efficiently inhibited, and the pulmonary and mediastinal metastasis have also been prevented. The survival of the cancer bearing mice was prolonged. The bio-imaging applications of this system and the mechanism of the metastasis prevention are ongoing.
\end{abstract}

Key words: Multifunctional nanoparticles, Photothermal Therapy, Multidrug Resistance, Co-therapy.

\section{Introduction}

Efficient suppression of tumor growth and metastatic prevention are the two main purposes of cancer therapy [1]. Traditional methods widely used in clinical cancer therapy are chemotherapy and radiotherapy, particular the chemotherapy which has been gotten much attention to enhance its tumor suppression efficiency. In chemotherapy, the bioavailability of the chemodrugs intimately connected with the efficiency of tumor growth suppression. By nanocrystalization of chemodrugs (by modern nanotechnology) and the enhanced permeation and retention (EPR) effect of tumor vessels, the bioavailability of some chemodrugs can be enhanced. And the tumor growth obtains a degree of control by administrating with nanomedicine [2]. Some nanomedicines, such as Genexol-PM and Abraxane, etc. are in the phase III clinical trials or have been launched to the market [3, 4]. The tumor growth, however, still cannot be totally suppressed by chemotherapy due to the drug resistance and metastatic inherence of tumor cells [5-9]. To enhance the suppression of tumor, co-therapy administrations provide a convenient and efficient 
way, which include gene-chemo co-therapy [10], chemo-radiation co-therapy $[11,12]$, and thermal-chemo co-therapy [13, 14]. Co-therapy combines the advantages of each therapeutic manner. Among them, only chemo-radiation co-therapy has been applied in clinical treatment, which still cannot suppress the tumor growth efficiently or reduce the side effects. The researches of other co-therapeutic manners are still ongoing.

Even through, chemo-thermal co-therapy has been proved to be an efficient method in cancer therapy [15]. The heat generated by the outer stimulus can make the blood in the tumor vessel take place coagulation. Combining with the anticancer drugs which are intracellular active, chemo-thermal co-therapy may play synergy therapeutic effects in cancer therapy. Among the numerous stimuli for heat generating, near infrared (NIR) laser which can trigger some metal NPs, such as gold nanorods, gold nanoshells, gold nanocages, $\mathrm{Cu}$ and $\mathrm{Pd}$ based NPs etc. via SPR effect or vibration of electron has been highlighted [16-22]. NIR-based PTT provides a harmless way for cancer thermal therapy. Furthermore, star-shape or "cluster"-shape gold-based nanosystems also exhibit excellent performance as NIR-based PTT carriers in cancer therapy [23, 24]. In addition, by combining with PTT, the anticancer performance of chemotherapy can be enhanced [25].

Besides, in order to guide the nanoparticles (NPs) to the tumor site, surface modification of NPs with targeting ligands, e.g. small molecules, peptide, aptamer, etc. has been applied $[19,26]$. As an alternative choice, which is different from the bio-receptors mediated tumor targeting; physical manners for NPs tumor targeting have been developed. Extra-magnetic field (MF) mediated tumor targeting has been highlighted in the past decade [27-30]. Magnetic NPs can be efficient targeted to the tumor site as well as the tumor microenvironment (which is also called stromal tumor component) [31]. The tumor microenvironment regulates tumor progression and the spread of cancer in the body $[32,33]$. And magnetic NPs can enhance the contrast in tumor magnetic resonance imaging (MRI). Kinds of magnetic NPs, including some hybrid magnetic NPs, have been developed for enhanced MRI [34, 35]. However, most of these nanosystems have solid and compact microstructures, drug loading is limited. In order to obtain a suitable theranostic nanocarrier for chemo-photothermal co-therapy, herein, we design a mesoporous magnetic gold "nanoclusters" (MMGNCs) as the carrier for chemo-photothermal therapy of breast cancer. Mesoporous structure is used for drug loading, magnetic property is used for extra-MF mediated tumor site targeting and MRI, and gold NPs decoration give MMGNCs with strong NIR adsorption. By extra-MF mediation, the MMGNCs can be targeted to the tumor site or tumor microenvironment. With the extra-NIR laser irradiation, chemo-photothermal co-therapy can be achieved, as shown in Scheme 1. In this work we mainly focus on the preparation, properties and in vivo anticancer performance of MMGNCs.

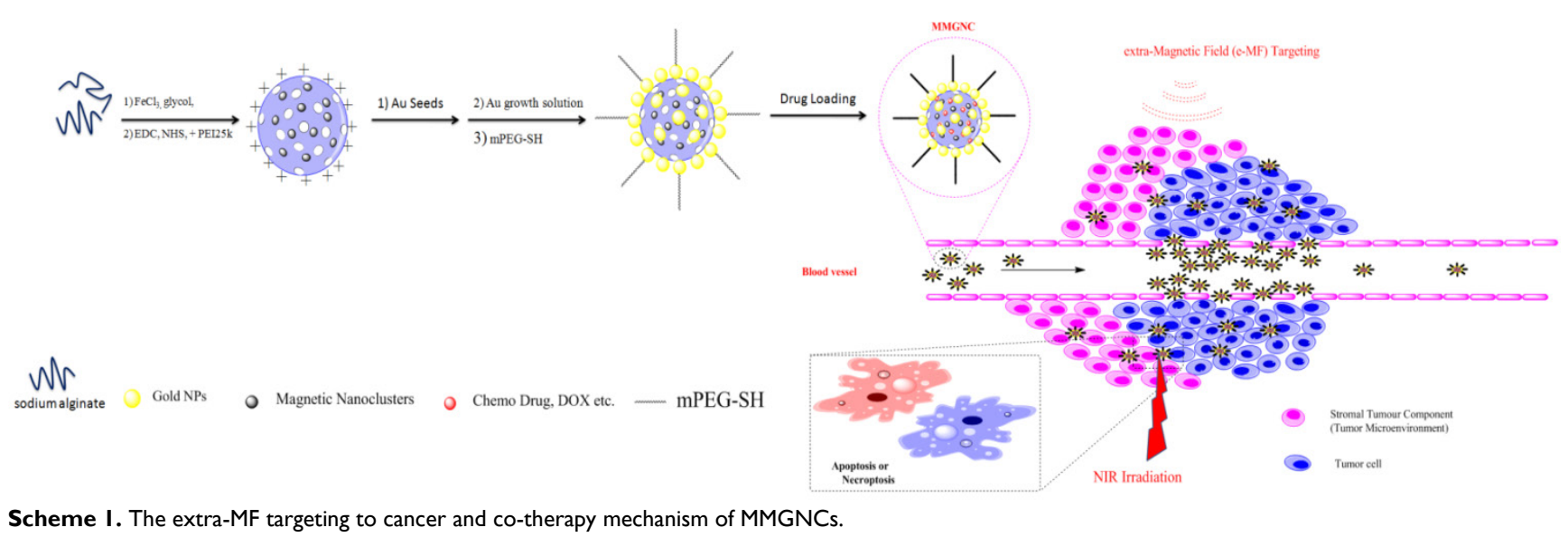

\section{Results and Discussion}

Preparation and characterization of MMGNCs. A two steps procedure (as illustrated in Scheme 1) was applied to prepare MMGNCs. We have firstly prepared the mesoporous precursor-mesoporous $\mathrm{Fe}_{3} \mathrm{O}_{4}$ nanoclusters (MFeNCs) (Figure 1a) by solvent thermal synthesis method [36-40]. From figure 1a, the uniform cluster structure of the NPs indicates that the MFeNCs have been successfully synthesized. Then, we prepared the MMGNCs by seed mediated growth method. From Figure $1 b$ and $c$, two different metal nanocrystals were found from the structure of the NPs. By HR-TEM observation (Figure 1d), two crystal lattice spacing (CLS) were measured, which were $0.4813 \mathrm{~nm}$ and $0.2417 \mathrm{~nm}$, corresponded to the CLS of 
$\mathrm{Fe}_{3} \mathrm{O}_{4}$ and $\mathrm{Au}$, respectively [41, 42], and combining with the $X$ ray diffraction testing (Figure 1e), it indicated that the Au NPs were successfully decorated on the surface of MFeNCs. The magnetic $\mathrm{Fe}_{3} \mathrm{O}_{4}$ structure was also confirmed by FTIR (Supplementary Material: Figure S1). By adjusting the dose of the $\mathrm{HAuCl} 4$ and the kinds of reductants, the number of the Au NPs decorated onto the surface of MFeNCs can be qualitatively controlled, as shown in figure $1 \mathrm{~b}$ and $1 \mathrm{c}$. MMGNCs can be well dispersed in water (Figure 1f). By DLS measurement, the average particle size of MMGNCs is $\sim 150 \mathrm{~nm}$ (Figure 1g), surface charge is $\sim-25 \mathrm{mV}$ which opposite to the MFeNCs $(+26 \mathrm{mV})$ (figure 1h). From the TEM images of the MMGNCs, porous structure was observed. By nitrogen adsorption-desorption measurements, we further confirmed the mesoporous structure of the MMGNCs and found that the number of the Au NPs can influence the pore size distribution of the MMGNCs. The pore sizes of the MFeNCs are mainly distributed in two regions which are $\sim 1.5 \mathrm{~nm}$ (small pore) and $\sim 10 \mathrm{~nm}$ (large pore), respectively, as shown in figure $1 \mathrm{i}$. While a few $\mathrm{Au}$ NPs were decorated onto the MFeNCs, the number of the large pores of MFeCs was decreased, while the small pores barely changed. While the Au NPs further increased, the number of the small pores decreased, and the large pores increased, as shown in figure 1i. The reason for the results is obvious. Form the TEM images, we can find that the particle size of the $\mathrm{Au}$ NPs is approximate $10 \mathrm{~nm}$. While the Au NPs are few, the large pores of the MFeNCs are embedded with Au NPs, which causes the decrease of the number of large pores. While the Au NPs are further increased, the MFeNCs are covered by Au NPs; however, the Au NPs are not forming a compact layer on the MFeNCs surface, which is forming a new porous structure on the outer layer of MMGNCs. The pore size of this layer is $\sim 10 \mathrm{~nm}$. Moreover, as the Au NPs increase, the small pores of the MFeNCs are covered by the $\mathrm{Au} \mathrm{NPs,}$ which decreases the number of the small pores. Even though, the change of the pore size distribution caused by the number of the Au NPs has little effect onto the surface area of MFeNCs and MMGNCs. The surface area of the MFeNCs, MMGNCs 1 and MMGNCs 2 were 45.94, 45.32 and $45.05 \mathrm{~m}^{2} / \mathrm{g}$, respectively, pore volume were 0.170 , 0.149 and $0.123 \mathrm{~cm}^{3} / \mathrm{g}$, respectively, and average pore size were 11.4, 8.7 and $6.8 \mathrm{~nm}$, respectively (Figure $2 a)$, it demonstrated that the mesoporous structure of MFeNCs changes a little after Au NPs decoration. To obtain expected drug loading capacity, we want to maintain the mesoporous structure, even after $\mathrm{Au}$ NPs decoration. Therefore, we do not hope to form a compact layer of gold on the surface. So we designed this gold "nanocluster" structure with magnetic properties to combine the application of photothermal and chemo co-therapy.

Photothermal Effect of MMGNCs. By Au NPs decoration, MMGNCs have strong absorption in NIR region. As shown in figure 2a, MMGNCs aqueous dispersion has much stronger NIR absorption than MFeNCs, and the intensity of the absorption increases as the number of the Au NPs increases (insets of figure 2a). The NIR absorption is the basic property in NIR-based PTT. By the extra-NIR laser irradiation, the temperature of the MMGNCs dispersion reached to $52{ }^{\circ} \mathrm{C}$ in $5 \mathrm{~min}$, which was much higher and faster than MFeNCs dispersion $\left(\sim 30^{\circ} \mathrm{C}\right.$ in $\left.5 \mathrm{~min}\right)$ and water (barely changed) (Figure 2b). It is more obvious from the infrared thermal images of the dispersion under NIR laser irradiation, as shown in figure 2c. Many studies have found that the cancer cell cannot survive while the environment temperature is higher than 42 ${ }^{\circ} \mathrm{C}$. MMGNCs exhibited its potential properties as a candidate for cancer photothermal therapy. Furthermore, after irradiation, the NIR absorption of MMGNCs barely changed, and its structure is stable (Figure 2d). For the number of the Au NPs has significant effect onto the NIR absorption of the MMGNCs, and the NIR absorption is directly connecting with the photothermal performance of the MMGNCs, considering the mesoporous structure for drug loading, the Fe and Au contents in MMGNCs which are chosen for the following studying are $\sim 33 \%$ and $\sim 15 \%$ (by EDX and ICP-AES measurement) respectively (Supplementary Material: Table S1).

Magnetic properties of MMGNCs. As magnetic NPs, the magnetization is another important property of MMGNCs. The saturation magnetization value of MMGNCs was $66.7 \mathrm{emu} /(\mathrm{g} \mathrm{Fe})$, which was lower than MFeNCs with $101.7 \mathrm{emu} /(\mathrm{g} \mathrm{Fe})$ (Figure 3a). It indicated that the decoration of $\mathrm{Au}$ NPs on the MFeNCs could weaken the magnetization of the NPs. Even through, MMGNCs still has appropriate magnetic properties. Moreover, because the blood in body vessel is continuously flowing, the research about the effect of the blood flowing velocity onto the magnetic NPs targeting onto the tumor sites under the extra-MF mediation is significance. It provides guidance for extra-MF targeting in vivo application. In here, we measured the in vitro extra-MF targeting performance of MMGNCs under extra-MF ( 0.2T) by blood vessel flowing simulation (Figure $3 b$ ). Under the velocity of $0.3 \mathrm{~cm} . \mathrm{s}^{-1}, \sim 60 \%$ of the MMGNCs were captured at the magnetic zone in $1 \mathrm{~h}$, and $\sim 85 \%$ in $2 \mathrm{~h}, \sim 95 \%$ in $3 \mathrm{~h}$, respectively. While the velocity increased to $3 \mathrm{~cm} \cdot{ }^{-1}$, the portion captured by the magnet was decreased, $\sim 35 \%$ in $1 \mathrm{~h}, \sim 68 \%$ in $2 \mathrm{~h}$, and $\sim 80 \%$ in $3 \mathrm{~h}$, respectively. The content of the NPs captured by the magnet is velocity dependent. Capillary vessels are widely 
distributed in tumor tissue, and the blood velocity in the capillary vessel is $\sim 0.3 \mathrm{~cm} . \mathrm{s}^{-1}$. It means that most of the MMGNCs could be captured in the tumor site by the extra-MF in $2 \mathrm{~h}$ if MMGNCs are moving in the capillary vessels of the tumor.

Drug loading and releasing behavior of MMGNCs. The loading efficiency of DOX loaded into MMGC NPs could reach to $80 \%$ and the drug loading capacity could reach to $15 \%$ (Figure 4 a) by equilibrium adsorption method. Besides, DOX released from the MMGNCs exhibited $\mathrm{pH}$ and laser irradiation stimuli dependence, respectively (Figure $4 b$ ). While the laser pulsed on, a burst release of DOX took place, which was much faster than that without laser irradiated (confirmed by the fluorescence spectrums of the release mediums, Figure 4c). While the laser on, the temperature of the medium surrounding the NPs increases, which increases the penetration of the water and the diffusion rate of the drug from the NPs to the medium. Furthermore, as the $\mathrm{pH}$ decreased, the amplitude of the DOX burst release triggered by NIR irradiation increased. The $\mathrm{pH}$ dependence of the drug release from the NPs is mainly due to the solubility of DOX increases while the $\mathrm{pH}$ value of the medium decreases. DOX released from MMGNCs is controllable by the mean of heat as well as $\mathrm{pH}$, which is similar to the report published by $\mathrm{Li}$ et al [43]. It is well known that the $\mathrm{pH}$ values of the tumor site and cytoplasm is lower than the body plasma. MMGNC is a promising drug delivery system for cancer therapy.

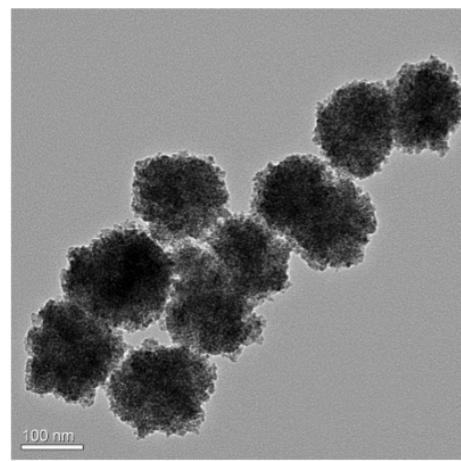

(a)

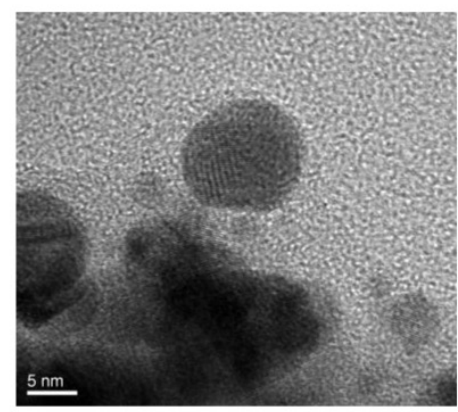

(d)

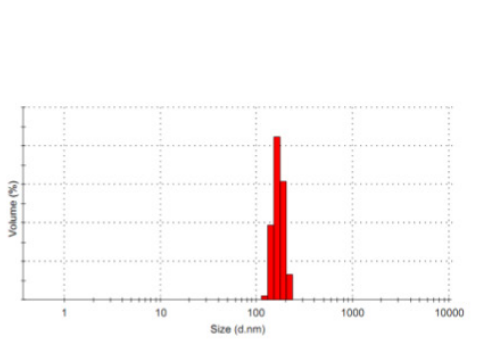

(g)

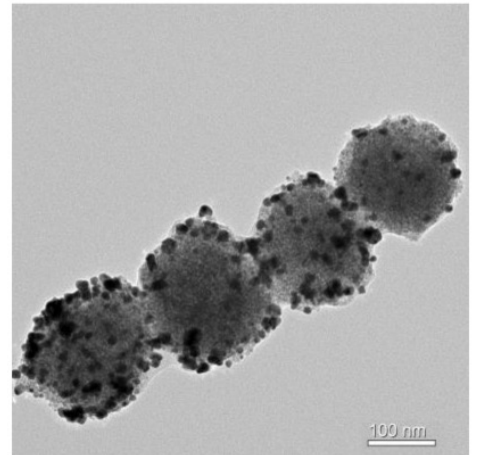

(b)

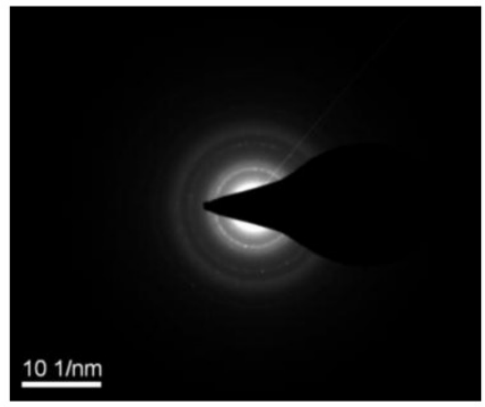

(e)

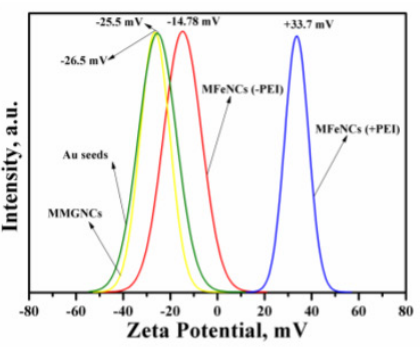

(h)

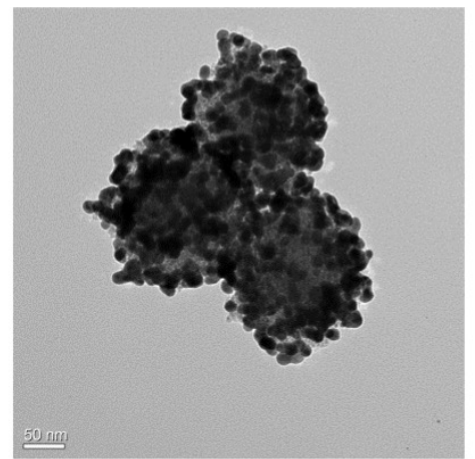

(c)

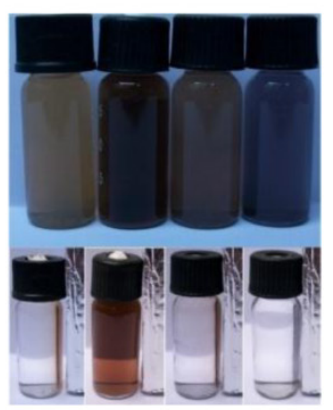

(f)

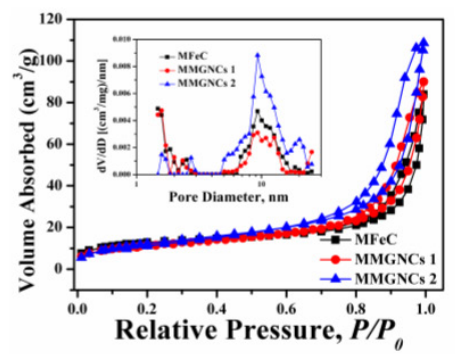

(i)

Figure I. Characterization of MMGNCs. TEM images of (a) MFeNCs, (b) MMGNCs I, (c) MMGNCs 2, (d) HR-TEM image of MMGNC, (e) X-ray diffraction image of MMGNCs, (f) the appearance of MMGNCs aqueous dispersion, (g) the particle size distribution of MMGNCs measured by DLS, (h) zeta potential of the particles measure by DLS, (i) Pore size distribution of MFeNCs and MMGNCs. 


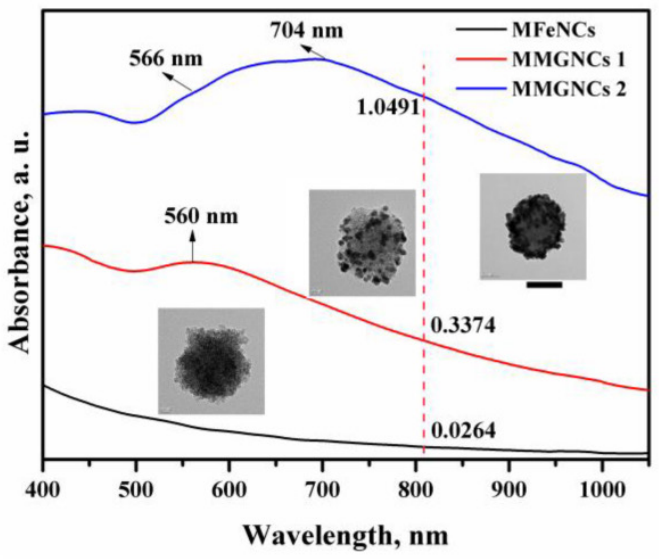

(a)

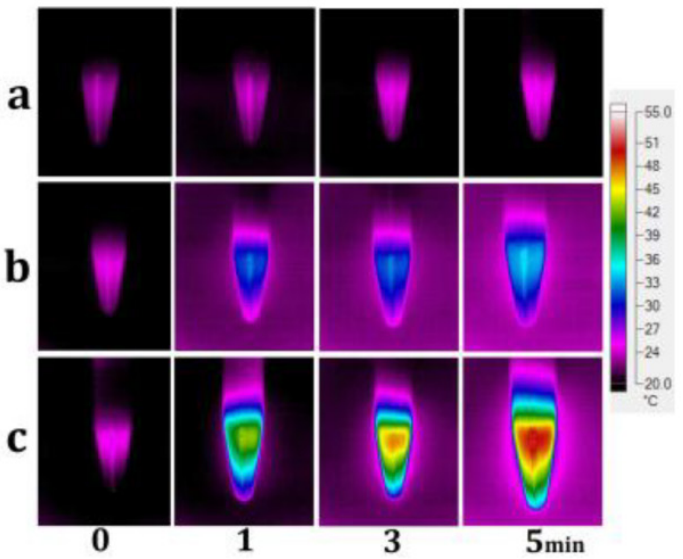

(c)

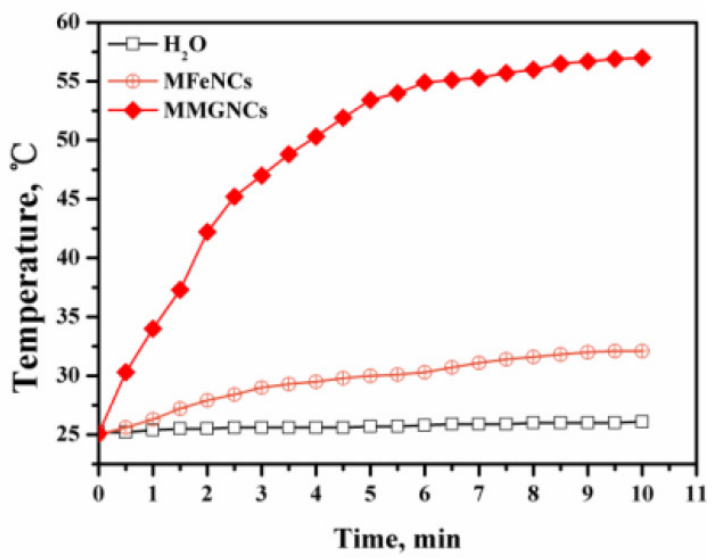

(b)

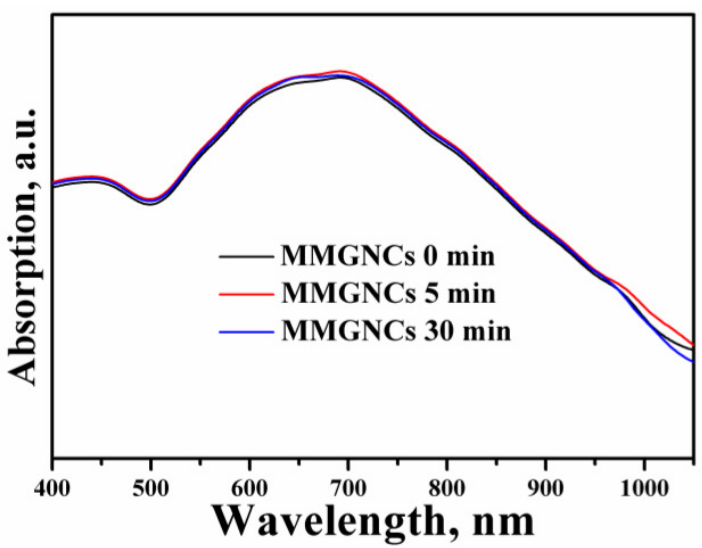

(d)

Figure 2. (a) UV-vis spectrums of MFeNCs and MMGNCs aqueous dispersion, Inset: TEM images of the correspond samples; (b) Relationships of the temperature of MMGNCs, MFeNCs dispersions and normal Saline and the $808 \mathrm{~nm}$ laser irradiation time, $70 \mu \mathrm{g} / \mathrm{mL}$; (c) infrared thermal images of the sample solution in EP tube under $808 \mathrm{~nm}$ laser irradiation; (d) Stability of MMGNCs under the irradiation of $808 \mathrm{~nm}$ laser. Laser intensity, $2.5 \mathrm{~W} / \mathrm{cm}^{2}$.

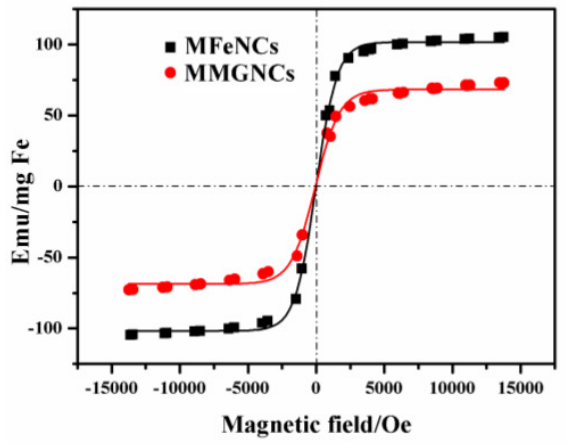

(a)

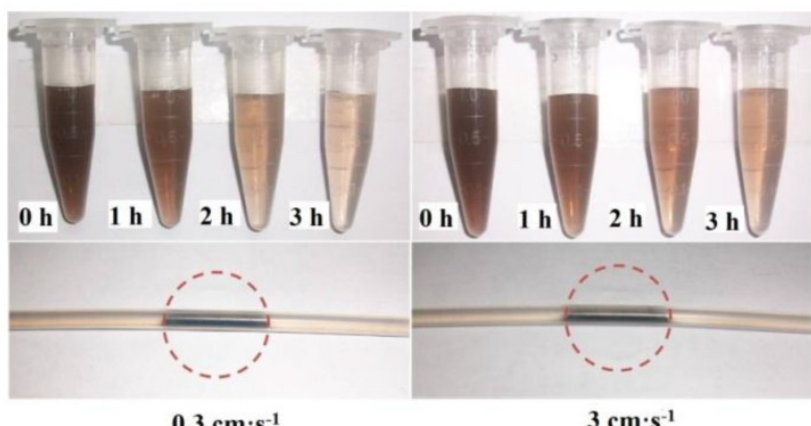

(b)

Figure 3. (a)The magnetic hysteresis curves of MFeNCs and MMGNCs. (b) MMGNCs retaining at different flow velocity. The red dash circle shows the magnet which fixed behind the paper. 


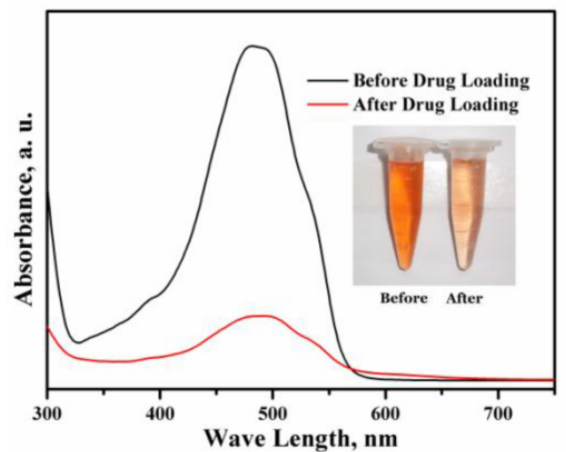

(a)
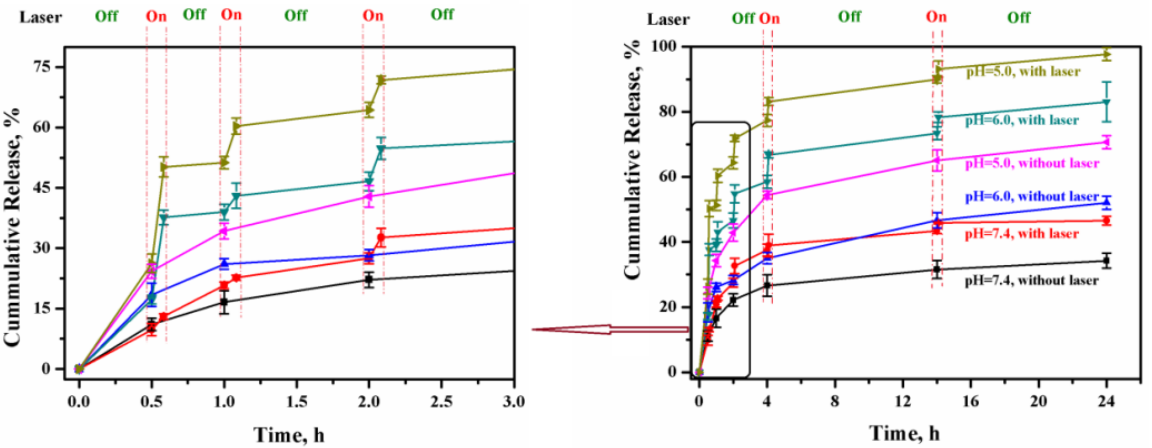

(b)

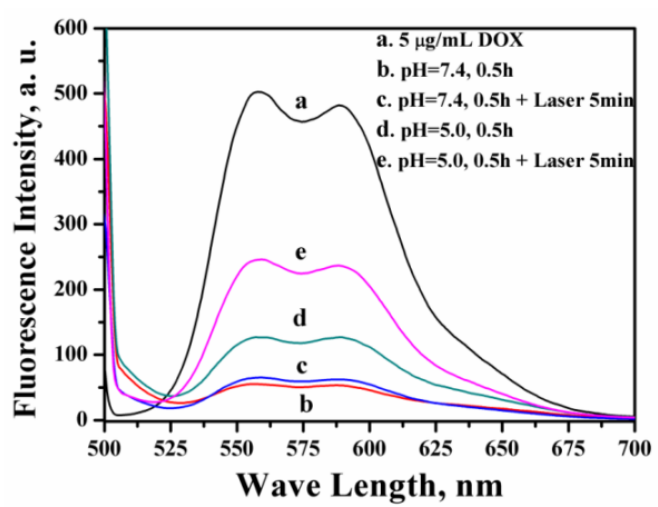

(c)

Figure 4. DOX loading (a) of MMGNCs and the release profiles (b) of DOX-loaded MMGNCs at different pH environment (with or without laser irradiation), (c) Fluorescence spectrum of the supernatants of the release mediums with and without the NIR irradiation during the release behavior studying of DOX-loaded MMGNCs.

Cytotoxicity of MMGNCs and DOX-loaded MMGNCs. Furthermore, no obvious cytotoxicity of blank MMGNCs to 293A cell lines were observed by MTT assays (Figure 5a). After Au NPs decoration, the surface charge of the NPs turned to negative, which decreased the MMGNCs cytotoxicity to the 293A cells. In order to evaluate the in vitro anticancer performance of MMGNCs as carrier for photothermal-chemo cotherapy, breast cancer cell-MCF-7 cells were chosen and extra $808 \mathrm{~nm}$ laser irradiation was introduced. To blank MMGNCs, after incubated with MCF-7 cells, under the NIR irradiation, as the irradiation time prolonging, the IC50 of blank MMGNCs decrease from $\sim 150 \mu \mathrm{g} / \mathrm{mL}$ ( 1 min irradiation) to $\sim 84$ $\mu \mathrm{g} / \mathrm{ml}$ ( $5 \mathrm{~min}$ irradiation) then to $\sim 68 \mu \mathrm{g} / \mathrm{mL}$ (10 $\mathrm{min}$ irradiation), as shown in figure $5 \mathrm{~b}$. By settling the MMGNCs concentration as $80 \mu \mathrm{g} / \mathrm{ml}$, different DOX content of DOX-loaded MMGNCs were used to evaluate the anticancer performance of DOX-loaded MMGNCs in vitro. The results are shown in figure $5 b$. The IC50 of DOX-loaded MMGNCs combining with 5 mins of laser irradiation $(0.84 \mu \mathrm{M})$ is lower than free DOX $(2.26 \mu \mathrm{M})$ or DOX-loaded MMGNCs without irradiation, indicating co-therapy exhibited more efficient tumor cells growth inhibition in vitro.

MR Imaging of MCF-7 Cells in vitro. After in- 
cubated with MMGNCs, the T2-weighted MRI images were obtained, as shown in figure 5c. A remarkable darkening appeared while the MMGNCs concentration was higher than $5 \mu \mathrm{g} / \mathrm{mL}$. While the MMGNCs concentration increased to $10 \mu \mathrm{g} / \mathrm{mL}$, the MRI gray level was decreased to $47 \%$. The results demonstrate that MMGNCs is promising candidate for tumor cells MR imaging.

In vivo Magnetic Targeting. Extra-magnetic field mediated specific site targeting has been proved as an efficient targeting manner. We have measured the extra-MF mediated tumor targeting performance of MMGNCs in vivo. After loaded with IR-820 (NIR fluorescence dye, loading capacity of MMGNCs to IR-820 was 7.6\%), IR-820 loaded MMGNCs were administrated to the $4 \mathrm{~T} 1$ breast cancer bearing balb/C mice. As shown in figure 6, with MF mediated targeting, the IR-820 fluorescence signal from the tumor site is much stronger than that without MF mediated. It indicates that by extra-MF mediation, much more MMGNCs are targeted to the tumor site. By semi-quantitatively calculation, the IR-820 fluorescence signal from the tumor which is undergoing extra-MF mediated targeting is by 1.6 factors of those without. Extra-MF mediated tumor targeting is an efficient targeting manner.

In vivo Photothermal Performance. From in vitro test, we found that MMGNCs has excellent photothermal performance. Infra-red thermal imag-

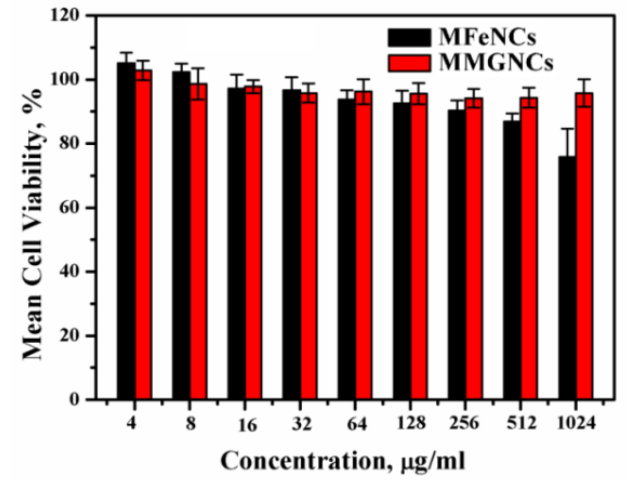

(a) ing was used to evaluate its photothermal performance in vivo. As shown in figure $7 \mathrm{a}$, with the extra-MF mediated tumor targeting for $4 \mathrm{~h}$ after MMGNCs was administrated, with the $808 \mathrm{~nm}$ laser irradiation for $1 \mathrm{~min}$, the tumor site temperature was increased to $49.3^{\circ} \mathrm{C}$ while that without extra-MF was $40.3^{\circ} \mathrm{C}$. And the tumor temperature of the mice which were not administrated with MMGNCs is barely increased. The results indicate that combining with the extra-MF mediated tumor targeting, MMGNCs is an efficient photothermal agent in vivo. By ICP-AES measurement, we found that the Fe and Au content of the tumors which were administrated with MMGNCs but without extra-MF exhibited no significant difference to those without MMGNCs administration (control groups), respectively. And the $\mathrm{Fe}$ and $\mathrm{Au}$ content of the tumors which were administrated with MMGNCs but combined with extra-MF are much higher than those without extra-MF, respectively, as shown in figure $7 \mathrm{~b}$. And the ratio of the increased $\mathrm{Fe}$ and $\mathrm{Au}$ content $(\mathrm{Fe} / \mathrm{Au})$ is almost equal to the $\mathrm{Fe} / \mathrm{Au}$ ratio of the MMGNCs used. It indicated that the enhanced photothermal performance of extra-MF mediated tumor targeting treated mice mainly ascribed to the enrichment of MMGNCs in the tumor site by extra-MF mediation. And from the $\mathrm{Fe} / \mathrm{Au}$ ratio results, it also demonstrated that the MMGNCs were stable in vivo.

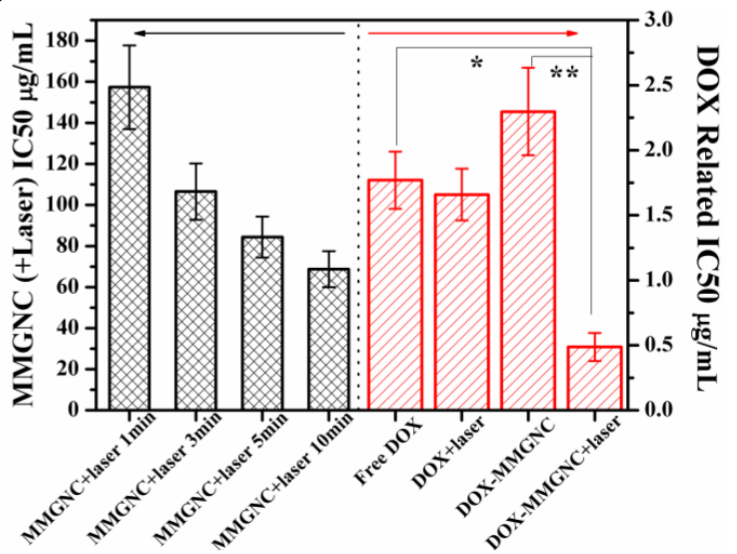

(b)

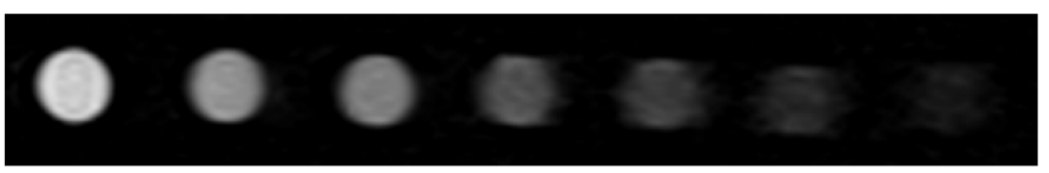

\begin{tabular}{|c|c|c|c|c|c|c|c|}
\hline MMGNCs & $\mathbf{0}$ & 1.25 & 2.5 & 5 & 10 & 20 & $40 \mu \mathrm{g} / \mathrm{mI}$ \\
\hline $\mathrm{Fe}$ & & 0.0075 & & & & & $0.24 \mathrm{mM}$ \\
\hline MRI gray & $\underline{100}$ & 92 & 83 & 72 & 47 & 24 & 6 \\
\hline
\end{tabular}

(c)

Figure 5. (a) Cytotoxicity of MFeNCs and MMGNCs to 293A cell lines. (b) cytotoxicity of MMGNCs+(808 nm laser irradiation) and DOX-loaded MMGC NPs to MCF-7 cell lines. (c) T2-weighted MRI images extracted from T2 measurements experiment (4.7 T; multiple spin-echo 2D imaging sequence; TR = I0000 ms; inter echo-time, $5 \mathrm{~ms}$; number of echo images, 256). 

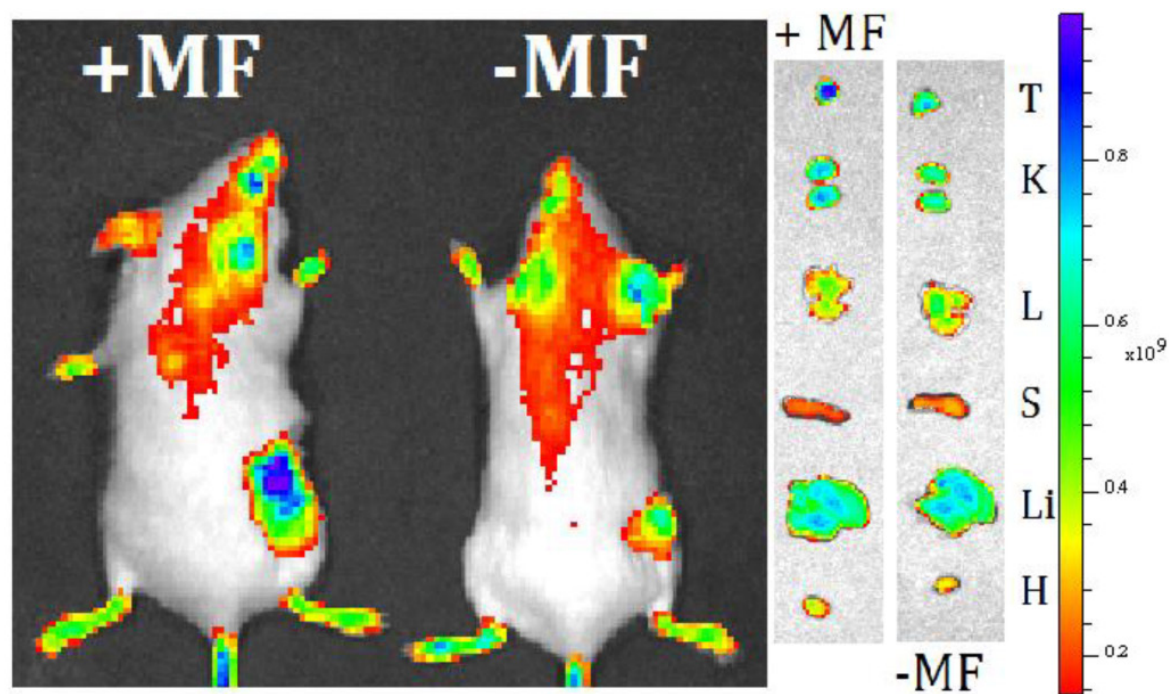

Figure 6. live imaging of 4TI breast cancer bearing mice treated with MMGNCs with extra-MF (+MF) and without extra-MF (-MF).
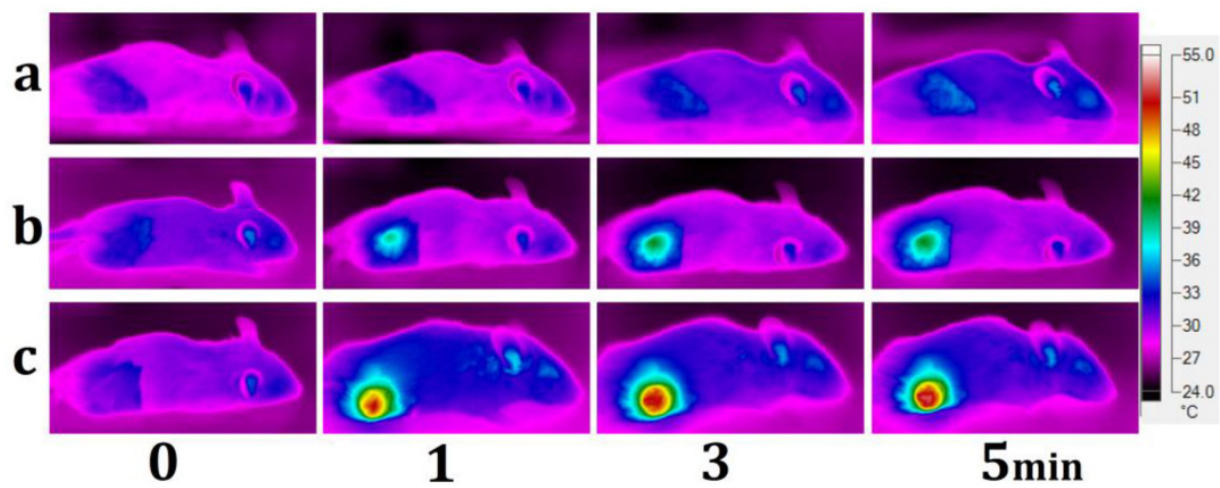

3

5 min

(A)

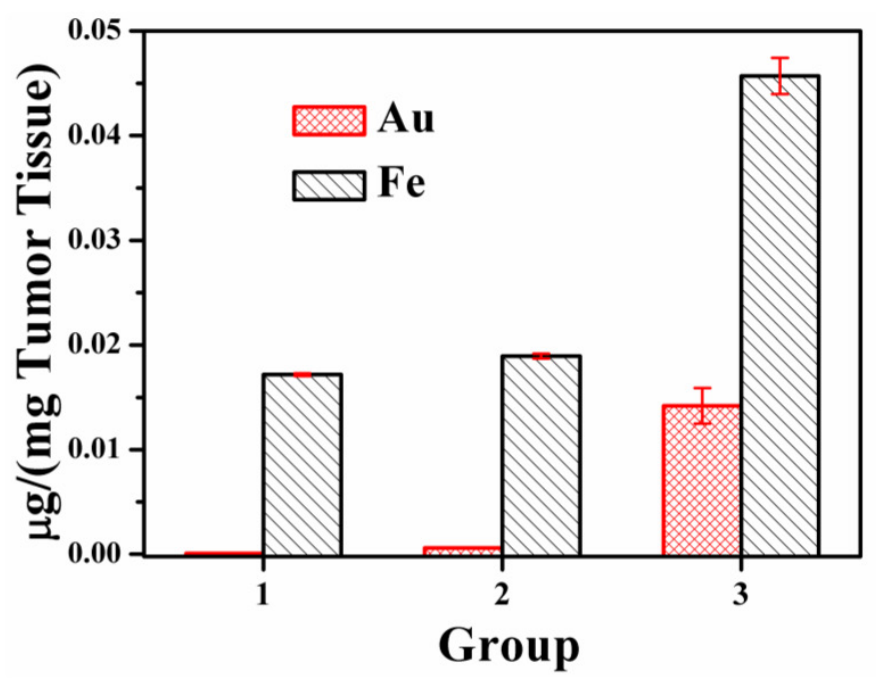

(B)

Figure 7. (A) the infrared thermal images of (a) NS treated mice, (b) MMGNCs treated without extra-MF mice, (c) MMGNCs treated with extra-MF mice; (B) the Au and $\mathrm{Fe}$ distributed in the tumor, Group I, the Au and Fe concentration in the tumor which was treated with normal saline; Group 2 and Group 3 are the Au and Fe concentration in the tumor which treated with MMGNCs without (group 2) and with (group 3) extra-MF mediated targeting, respectively. $\mathrm{p}<0.05$. 
In vivo therapy of $4 \mathrm{~T} 1$ breast cancer bearing Balb/C mice of DOX-loaded MMGNCs. We have chosen PTX and DOX as the model drugs in breast cancer therapy in vivo. The tumor inhibition in vivo (Figures 8a, b and c) and histopathological studying (Figure 8d) results revealed DOX-loaded MMGNCs had better anticancer performance than PTX-loaded MMGNCs. The lower anticancer efficiency of PTX-loaded MMGNCs may ascribe to the lower stability of the drug loading system and the thermal induced degradation of PTX [44]. After this drug selection by preliminary experiment, we used DOX-loaded MMGNCs for deeper research. 10 groups of tumor bearing Balb/ $\mathrm{C}$ female mice were treated by veil injection with Normal Saline (B), NS + laser (C), MMGNCs + laser $(D)$, MMGNC + MF + laser $(E, F)$, MMGNCs + DOX $(G)$, MMGNCs + DOX + MF $(H)$, MMGNCs + DOX + laser (I), MMGNCs + DOX + MF + laser $(J)$, free DOX with single administration $(K)$, and free DOX with four administrations group $(\boldsymbol{L})$, separately. By a single treatment with low DOX dosage (2.5mg DOX/Kg mouse weight), the tumor of the

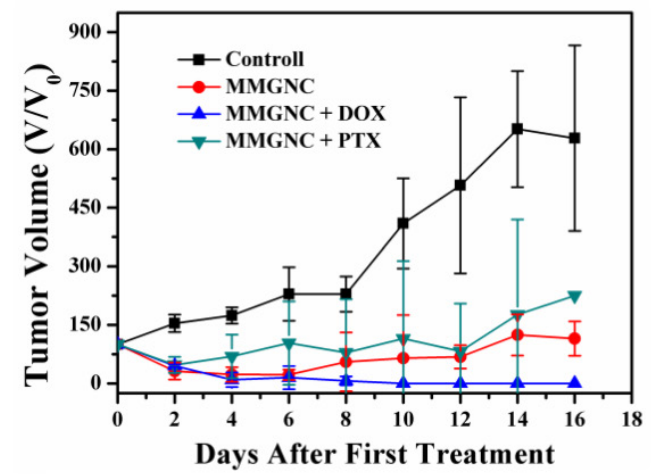

(a)

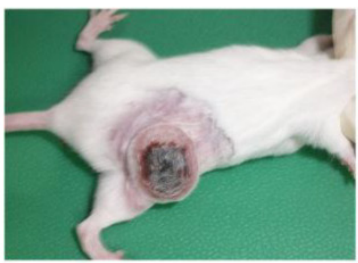

NS

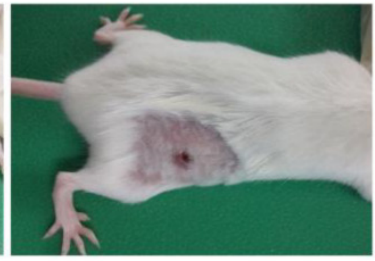

MMGNC

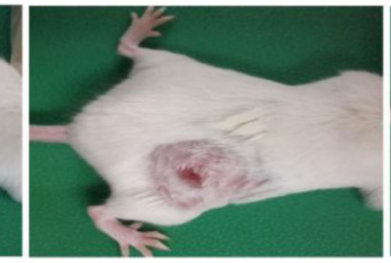

MMGNC+DOX

mice treated with $J$ is diminished (Figure 9a). It is much clear from the mouse photos took from different groups (Figure $9 b$ ). Although the tumor of the $\boldsymbol{F}$ treated mice also diminished at the preliminary stage, recurrence took place in 20 days after treatment. Under PTT, the groups with extra-MF are more efficiency in tumor inhibition than those without (while compared $\boldsymbol{F}$ and $\boldsymbol{J}$ with $\boldsymbol{D}$ and $\boldsymbol{I}$, respectively). The therapeutic effects of NS group has no significant difference with $C$ group, free DOX with one single dosage group (data did not show), $\boldsymbol{G}$ group and $\boldsymbol{H}$ group, for the DOX is injected by one single dosage (and the dosage is low) which hardly suppressed the growth of the tumor, and the group treated with $D$ is similar to the $I$ treated group which laser displaying the main role. For DOX was single administration, and the dosage was low, the injection of MMGNC or MMGNC+DOX has little side effect onto the organ, no significant weight variation is observed (Figure 9c), and the heart cytotoxicity of DOX is hardly observed (Supplementary Material: Figure S2).

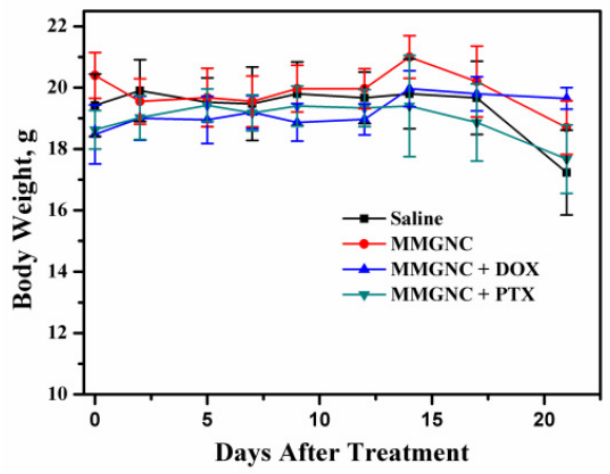

(b)

(c)

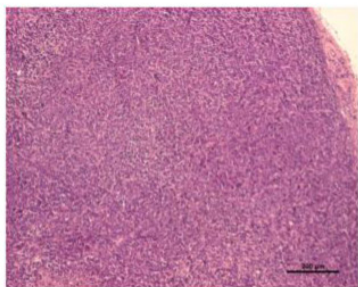

NS

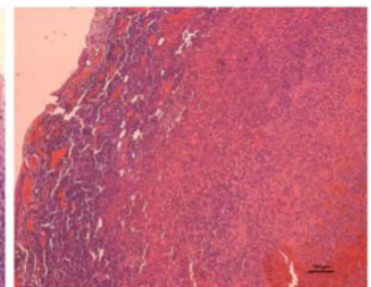

MMGNC

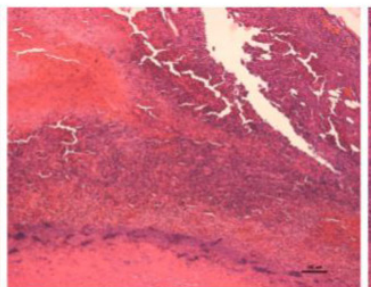

MMGNC+DOX

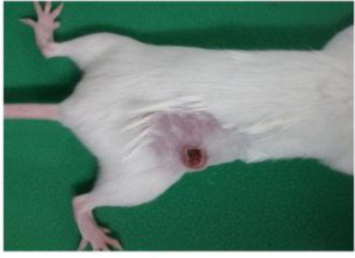

MMGNC+PTX

(d)

Figure 8. The anticancer performance of MMGNCs while loading with different model drugs. (a) the tumor volume variation of the mice with different treatments, (b) the body weight variation, (c) the digital photos of the mice with different treatments, (d) HE staining of the tumor samples in the 8 days after the first treatment. 


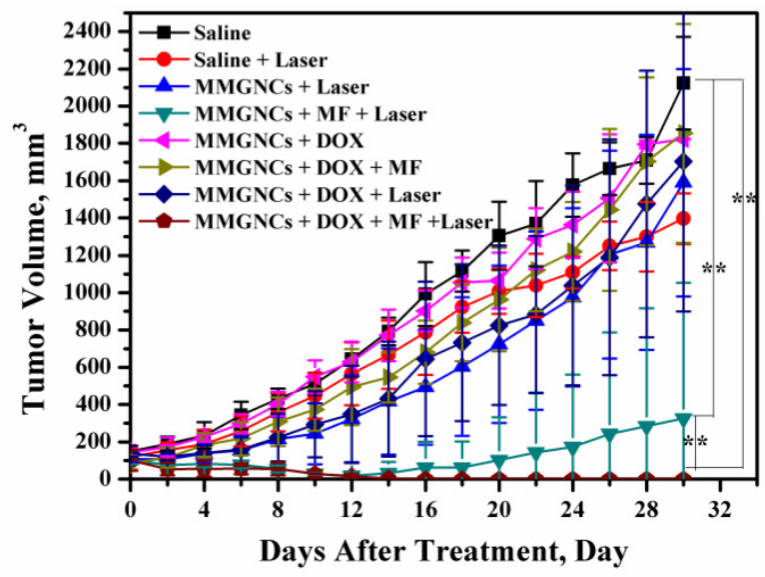

(a)
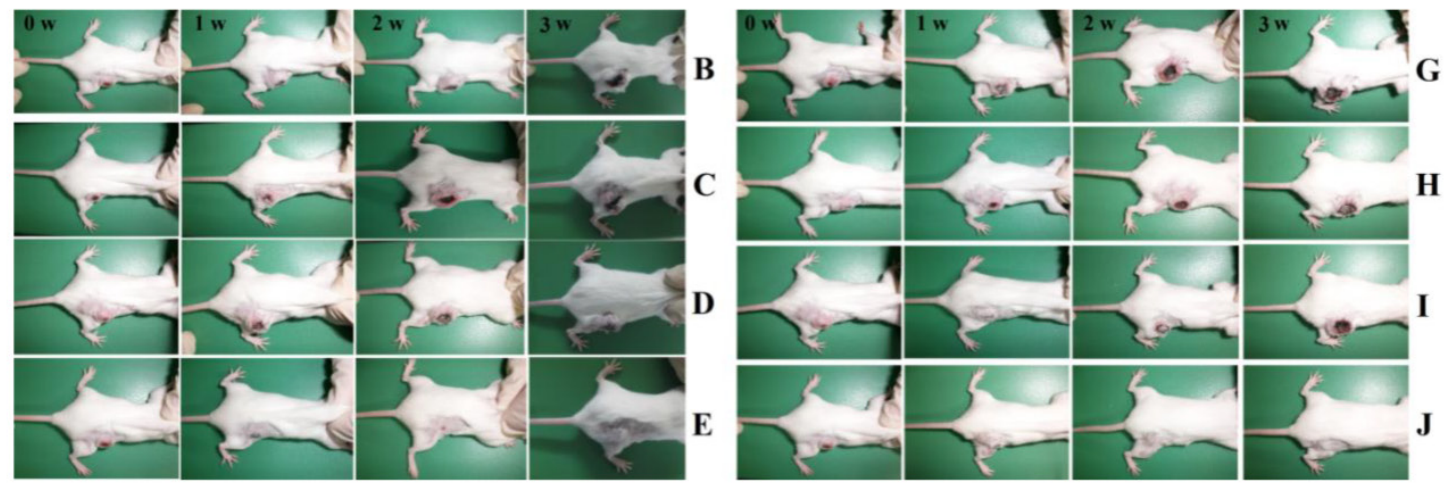

(b)

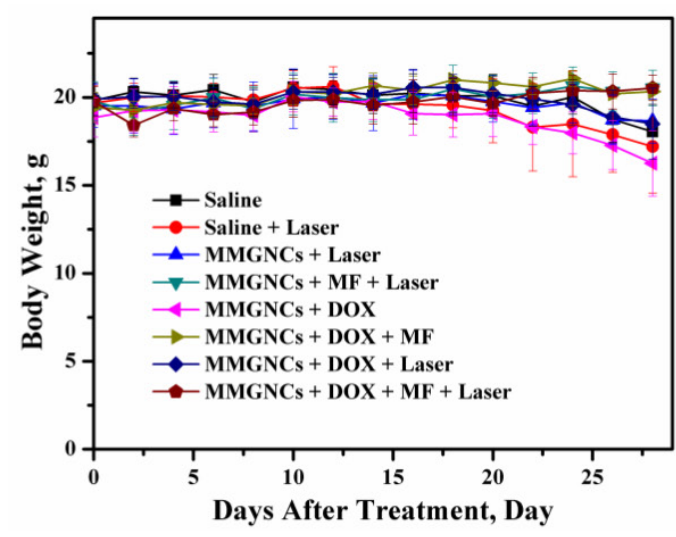

(c)

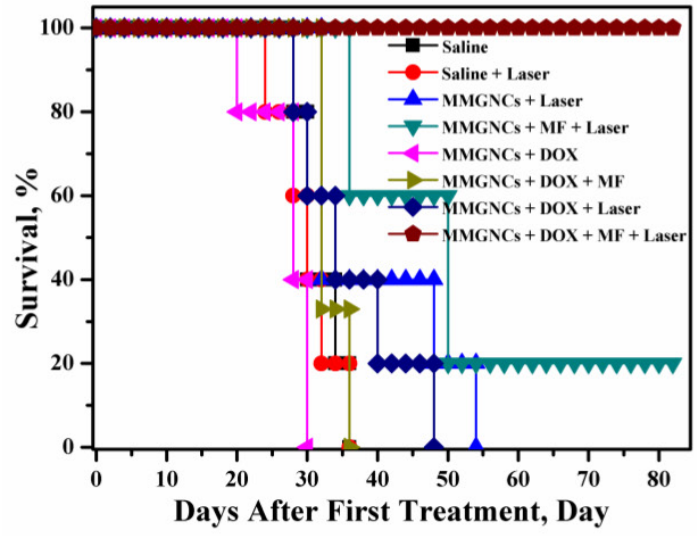

(d)

Figure 9. variation of (a) the tumor volume of the mice treated with different treatments. (b) Photo images of mouse in different groups after treated (photos were took at 0 weeks, I weeks, 2 weeks and 3 weeks after treated) (A, heath mouse; B, Normal Saline groups; $\boldsymbol{C}$, NS + laser groups; $\boldsymbol{D}$, MMGNCs + laser groups; $\boldsymbol{E}$, MMGNC + MF + laser (recurrence) samples; $\boldsymbol{F}$. MMGNCs + MF + laser samples; $G$, MMGNCs + DOX groups; $\boldsymbol{H}$, MMGNCs + DOX + MF groups; $\boldsymbol{I}$, MMGNCs + DOX + laser groups; J, MMGNCs + DOX + MF + laser groups). (c) and (d), variation of (c) body weight and (d) survival of the mice treated with different treatments.

All the mice treated with $J$ treated survived, and the $\boldsymbol{F}$ group had $20 \%$ chances to survive (Figure $9 \mathrm{~d}$ ). Treated with $D$ and $I$ have a chance to prolong the life cycle. And free DOX, $G$ treated mice all died much faster than NS groups which is cause by the heart cytotoxicity of DOX [45].

From the lungs and mediastinum tissues obtained from different groups by scarified the mouse in 26 days after first treatment, clearly tumor prolifera- tion (Figure 10a) and even some mediastinal metastasis (Figure 10b) took place in all treated groups except the groups treated by $J$, and the groups treated by $F$ has a chance to avoid the tumor proliferation. This is further confirmed by the HE staining of these organ samples (Figure 10c \& d).

The mechanism of the tumor proliferation and mediastinal metastasis inhibition of MMGNCs+DOX+MF+laser is complicated and still 
not clear yet. However, we guess it may have some connection with the change of the microenvironment around the tumor or the reinforcement of the anti-cancer immunity of the treated mice. By histology study of the tumor pathological section after treatments (the tumor tissues were obtained in the next day after the first treatment), we found that although apophtosis and necroptosis took place in the tumor site of the group treated by $J$ (Figure 11), there were still many tumor cells survived in the tumor site. But in the seven days after the first treatment, most of the tumor cells disappeared in the tumor site (Figure 8d). It may ascribe to the microenvironment around the tumor have been changed or the antiangiogenic took place after the photothermal chemo co-therapy by MMGNCs. However, the metastatic prevention of the treated mice in a long period may mainly ascribe to the anti-cancer immunity of the treated mice has been enhanced. We are still working on the connection between the anti-cancer immunity and the metastatic inhibition of the treated mice after the chemo-photothermal co-therapy.
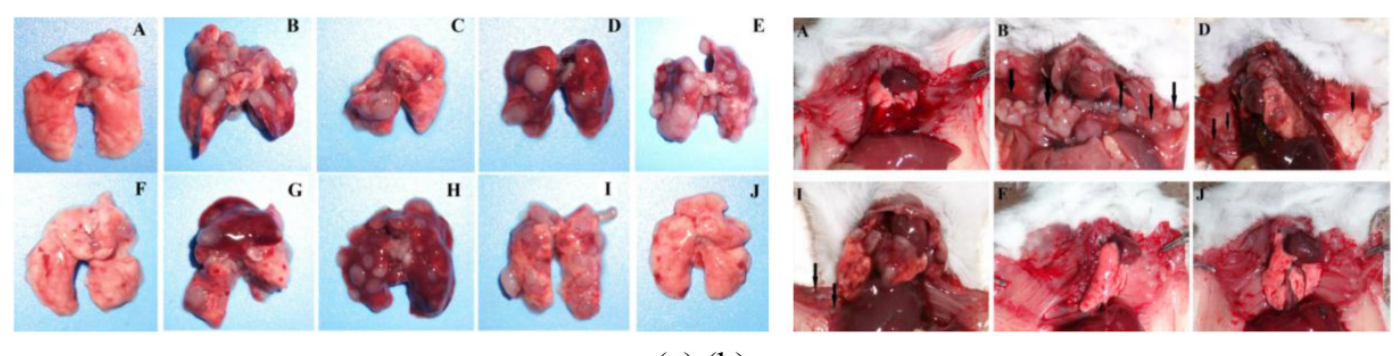

(a) (b)

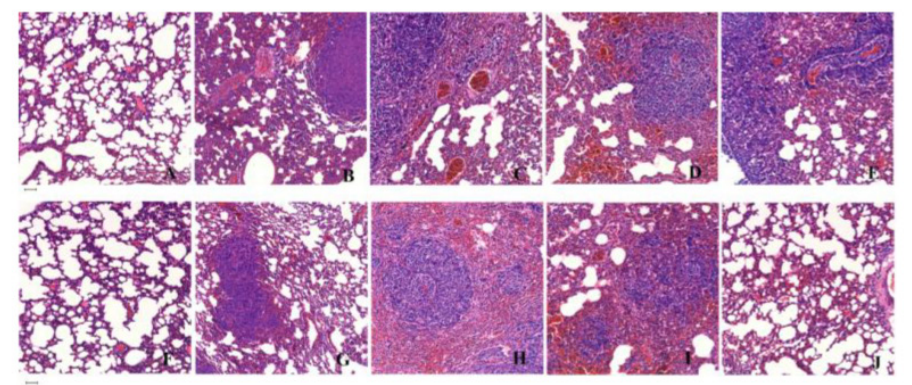

(c)

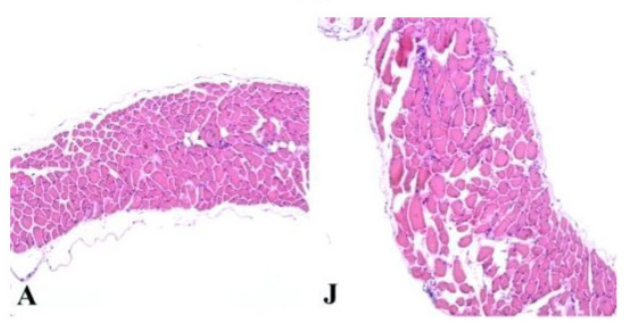

(d)

Figure 10. (a) lung tissues obtain from different groups after treatment, (b) Photographs of the mice in different groups after sacrificed. HE staining of the lung tissues (c) and mediastinum (d) obtained from the mice with different treatments, scale bar $=50 \mu \mathrm{m}$. It is clearly seen that the combination of extra-MF targeting, photothermal and chemo therapy could efficiently inhibit the happening of tumor pulmonary metastasis and mediastinal metastasis. (The capital letter marks corresponding to Figure 5).

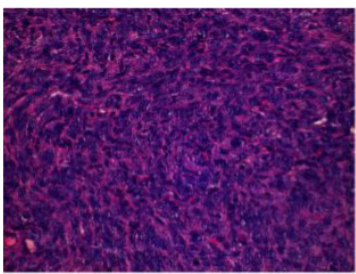

A

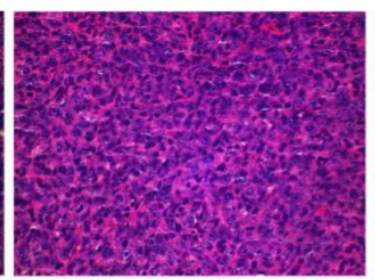

B

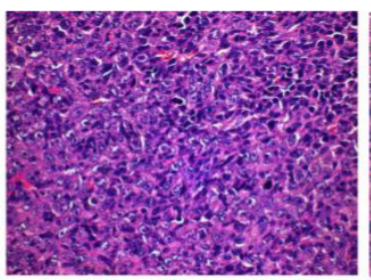

C

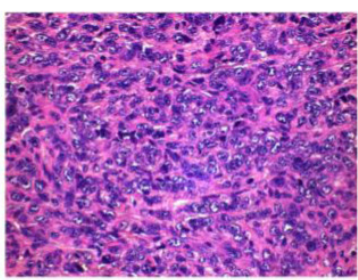

D

Figure I I. Histological observation of treated and untreated 4TI tumor tissues visualized using H\&E staining. (A) NS treated groups, (B) MMGNCs+Laser, (C) MMGNCs+MF+Laser, (D) MMGNCs+MF+DOX+Laser. 


\section{Conclusion}

In summary, we have successfully designed and prepared a novel mesoporous magnetic gold "cluster" as carrier for chemo-photothermal co-therapy. By in vitro cytotoxicity testing, we revealed the combination of photothermal therapy and chemotherapy could cause more damage than chemotherapy or photothermal therapy did alone. And MMGNC exhibited extra-MF mediated tumor targeting. Efficient tumor growth inhibition and pulmonary and mediastinal metastasis prevention were obtained simultaneously by MMGNCs combined with extra-MF targeting and photothermal- chemo co-therapy. The cancer bearing mice survival was prolonged. The bio-imaging of this system and the mechanism of metastasis prevention investigations are ongoing.

\section{Materials and Methods}

Materials. All chemicals involved in this work were analytical grade and used without further purification. Sodium alginate, Ethylene glycol, PEI25K, EDC, NHS, 3-(4,5-dimethylthiazol-2-yl)-2,5-diphenyl tetrazolium bromide (MTT), IR-820 and mPEG-SH were purchased from Sigma-Aldrich and used as received. $\mathrm{HAuCl}_{4}, \mathrm{~K}_{2} \mathrm{CO}_{3}$, and $\mathrm{NH}_{4} \mathrm{COOH}$ were purchased from Sinopharm Chemical Reagent Co. Ltd, Shanghai, China. $\mathrm{FeCl}_{3} \cdot 6 \mathrm{H}_{2} \mathrm{O}$ and Doxorubicin chloride was obtained from Zhejiang Hisun Pharmaceutical Company, Zhejiang, China.

\section{Synthesis of MMGNCs}

Synthesis of MFeNCs. Mesoporous $\mathrm{Fe}_{3} \mathrm{O}_{4}$ nanoclusters (MFeNCs) were prepared through a modified solvothermal reaction: $1.35 \mathrm{~g}$ of $5 \mathrm{mmol}$ $\mathrm{FeCl}_{3} .6 \mathrm{H}_{2} \mathrm{O}, 3.85 \mathrm{~g}$ of $0.05 \mathrm{~mol} \mathrm{NH}_{4} \mathrm{COOH}$, and $0.50 \mathrm{~g}$ of sodium alginate were dissolved in $70 \mathrm{~mL}$ of ethylene glycol. The mixture was stirred vigorously for 1 $\mathrm{h}$ at $160^{\circ} \mathrm{C}$ under the protection of N2 to form a homogeneous brownish solution, then $0.125 \mathrm{~g}$ of PEI25K was added to this solution, followed with $12 \mathrm{mg}$ of EDC and $6 \mathrm{mg}$ of NHS, and reacted for another $1 \mathrm{~h}$, and then transferred the solution into a Teflon-lined stainless-steel autoclave ( $100 \mathrm{~mL}$ capacity). The autoclave was heated to $200^{\circ} \mathrm{C}$ and maintained for $12.5 \mathrm{~h}$. Then it was cooled to room temperature. The black MFeNCs were then rinsed several times with ethanol and water alternately under ultrasonic conditions. The resulted precipitation was dried in vacuum.

Preparation of MMGNCs. The MMGNCs was prepared though a gold seeds mediated growth reaction with some modification.

Preparation of $A u$ seeds. Au seeds were synthesized according to the report of Edwards et al. [46]. In briefly, $12 \mathrm{ml}$ of $80 \%$ tetrakis-hydroxymethyl -phosphonium chloride (THPC) and $0.25 \mathrm{ml}$ of $2 \mathrm{M}$ $\mathrm{NaOH}$ were added to $45 \mathrm{ml}$ of DI water. The mixturewas stirred vigorously for $5 \mathrm{~min}$ before $2 \mathrm{ml}$ of $1 \%$ $\mathrm{HAuCl}_{4}$ was quickly introduced in one shot. An immediate color change to dark brown was observed. The solution was stored in a lightproof container at 4 ${ }^{\circ} \mathrm{C}$ for 1 week before used.

Growth of gold NPs. Au seeds (1 3nm) were first attached to MFeNCs by mixing $1 \mathrm{ml}$ MFeNCs solution ( $25 \mathrm{mg} / \mathrm{ml} \mathrm{MMC}$ ) with $5 \mathrm{ml}$ of Au seed solution. After stirring at room temperature for $4 \mathrm{~h}$, excess Au seeds were removed by centrifugation at $8000 \mathrm{rpm}$ and repeated washing with water. In growth solution preparation, $1.5 \mathrm{ml}$ of $1 \% \mathrm{HAuCl}_{4}$ was added into $100 \mathrm{ml}$ of DI water containing $25 \mathrm{mg}$ of potassium carbonate. The solution turned colorless over a course of $30 \mathrm{~min}$. The solution was aged for one day in $4{ }^{\circ} \mathrm{C}$ before use. Under vigorously stirring, $10 \mathrm{ml}$ of MFeNCs (2.5 $\mathrm{mg} / \mathrm{ml} \mathrm{MFeNCs}$ ) solution (with Au seeds attached) was added to the aged Au growth solution. Then, a $100 \mu \mathrm{l}$ of reducing agent (formaldehyde, $38 \sim 40 \%$ ) was introduced drop-wisely. The color of the mixture immediately changed from light-brownish to brownish-blue in $10 \mathrm{~min}$. mPEG-SH was attached to the particles to stabilize the MMGNCs. The number of the $\mathrm{Au}$ NPs decorated onto the MMGNCs can be adjusted by changing the Au seed solution or growth solution dosages.

\section{Characterization of MMGNCs}

Micro-morphology. The morphology of the prepared samples was observed under a transmission electron microscope (TEM) (Tecnai G2 F20 S-TWIN, FEI, American): the samples were dispersed in distilled water and placed on a copper grid covered with nitrocellulose.

Chemical structure. Fourier transform infrared (FT-IR) analyses of the freeze-dried samples were performed on a Nicolet 6700 FTIR spectrometer (Thermo Scientific) to identify their chemical structures. Transmission infrared spectra were obtained in the wavenumber range of $400-4000 \mathrm{~cm}^{-1}$. The samples were dried and mixed with $\mathrm{KBr}$ to be compressed to a plate for measurement.

Particle size and surface charge. The zeta potentials of the samples were measured by MALVEN dynamic laser scattering (DLS).

NIR adsorption. The NIR adsorption of MMGNCs aqueous dispersions were measured by UV-vis spectrophotometer (PekinElmer, Lambda 35 UV-vis spectrometer).

Surface area, pore volume and pore size distribution. The surface area, pore volume and pore size distribution of the samples were tested by Nitrogen adsorption-desorption measurements. Nitrogen adsorp- 
tion-desorption measurements were performed on an ASAP2020 (Micromeritics, USA) accelerated surface area analyzer at $77 \mathrm{~K}$. Before measuring, the samples were degassed in a vacuum at $120^{\circ} \mathrm{C}$ for at least $6 \mathrm{~h}$.

Photothermal effect. MMGNCs aqueous dispersion $(\sim 70 \mu \mathrm{g} / \mathrm{mL})$ was irradiated by $808 \mathrm{~nm}$ laser, and the temperature of the dispersion was recorded by portable electronic thermometer with a probe.

Magnetic hysteresis. Magnetic characterization was carried out with a vibrating sample magnetometer on a model 6000 physical property measurement system (Quantum Design, USA) at $300 \mathrm{~K}$.

Composition of MMGNCs. Elemental Analyser. The $\mathrm{C}, \mathrm{H}, \mathrm{N}$, S element content in the samples were carried out by an Elemental Analyser (Euro EA 3000, Leeman Labs INC., USA). Energy Dispersive X-Ray Spectroscopy (EDS) The surface element composition of the samples were obtained from EDS measurement via SEM (JSM-7500F, JEOL, Japan). ICP-AES. The Fe and Au contents of MMGNCs were measured by Inductively Coupled Plasma-Atomic Emission Spectrometer (ICP-AES, SPECTROARCOS, Spectro, Germany). The samples were dissolved by digestio digestant (HNO3 $: \mathrm{HCl}: \mathrm{HPO} 4=3: 1: 2$ ).

Drug loading and releasing behavior studying of MMGNCs. DOX was loaded into the MMGNCs by equilibrium adsorption method. Briefly, $15 \mathrm{mg}$ of MMGNCs were first dispersed in $5 \mathrm{ml}$ of water, then mixed with $1 \mathrm{ml}$ of DOX solution (vary concentration) for $24 \mathrm{~h}$ in a shaker under the speed of $120 \mathrm{rpm}$. Then the DOX-loaded MMGNCs were separated by centrifugation and washed by PBS $(\mathrm{pH}$ value $=7.4)$ for 5 times. And the drug-loaded NPs were lyophilized for further use.

The DOX-loaded MMGNCs release performance was performed by dialysis method with different medium ( $\mathrm{pH}=5.0,6.0,7.4$, separately). $5 \mathrm{mg}$ of DOX-loaded MMGNCs (loading capacity was $10.2 \%$ ), were first redispersed in $2 \mathrm{ml}$ of PBS, then transferred it into a dialysis bag with the cutting off molecular weight of 8000 14000, and sealed the bags then placed into $8 \mathrm{ml}$ of releasing medium (different $\mathrm{pH}$ value, separately), at pre-determined time, the releasing medium were replaced by the blank medium. And at pre-determined time point, the suspension were irradiated by $808 \mathrm{~nm}$ laser for $5 \mathrm{~min}$, and after the irradiation, the releasing medium was piped out immediately, and replaced by the blank releasing medium. The concentrations of the releasing mediums were measured by UV-vis spectrophotometer, and the cumulative released drug was calculated by the calibrated curve of DOX absorption in UV-vis spectrum at $490 \mathrm{~nm}$.

\section{Cytotoxicity of MMGNCs and DOX-loaded MMGNCs}

Cytotoxicity of MMGNCs to 2937 cells. The cytotoxicity of MMGNCs to the 293A cells were evaluated by MTT assay. The cells were cultured in a 5-95\% $\mathrm{CO}_{2}-\mathrm{O}_{2}$ atmosphere at $37 \mathrm{C}$. Cells were seeded on 96-well plates at a density of $10^{4}$ cells/well in their usual culture media, supplemented with $10 \%$ FBS and $1 \%$ PS. After $24 \mathrm{~h}$, the media without FBS were used to replace the usual ones and the cells were exposed to different concentration of MMGNCs for $24 \mathrm{~h}$, and then $100 \mathrm{uL}$ of MTT ( $5 \mathrm{mg} \mathrm{mL}-1)$ solution was added to each well and incubated at $37 \mathrm{C}$ for $4 \mathrm{~h}$. After the removal of the supernatants, $650 \mu \mathrm{L}$ of dimethyl sulfoxide (DMSO) was added to each well to dissolve the blue formazan crystal, and then the solution was transferred to the 96-well plates. The absorbance of the contents of each well was measured at $570 \mathrm{~nm}$ using an ELISA microplate reader (Bio-Rad). A mean value was obtained from the measurement of five test runs.

Cytotoxicity of DOX-loaded MMGNCs to tumor cells. The cytotoxicity of DOX-loaded MMGNCs to MCF-7 was evaluated as the same method used in MMGNCs cytotoxicity test to 293A cell lines with some modification. Laser was introduced into the measument. After incubated with DOX-loaded MMGNCs for $4 \mathrm{~h}$, the MCF-7 cells in each well were irradiated by $808 \mathrm{~nm}$ laser at the power density of 2.5 $\mathrm{W} / \mathrm{cm}^{2}$ for $5 \mathrm{~min}$. Then the cells were incubated for another $36 \mathrm{~h}$. The MTT addition is the same as the MMGNCs cytotoxicity to 293A testing.

in vitro vessel blood flowing simulation. MMGNCs aqueous dispersion flowed in a plastic tube with the diameter of $2 \mathrm{~mm}$ with the impetus driven by a peristaltic pump, while it reaches to the magnetic field region (dash circle); MMGNCs were captured by the magnet. And the dispersions were flowing circularly. At predetermined time, dispersion samples were collected to measure the concentration of MMGNCs. And the MMGNCs concentration was measured by UV-vis spectrum, and calculated by the linear fitting function obtained from the absorbance at $808 \mathrm{~nm}$ and the concentration of MMGNCs (Standard curve function, $y=0.00505 x+0.00414, R^{2}=0.99953, y$ is the adsorption value of the MMGNC dispersion at 808 $\mathrm{nm} ; \mathrm{x}$ is the concentration of MMGNCs dispersion, $\mu \mathrm{g} / \mathrm{mL}$ ) .

MR Imaging of MCF-7 Cells in vitro. MCF-7 cells were incubated with MMGNCs dispersion, after 24h incubation, the MCF-7 cells were washed by PBS $(\mathrm{pH}=7.4)$ for 3 times. Then the T2-weighted MRI images were extracted by T2 measurements experiment (7.0T, BioSpec®In-vivo MR Spectroscopy/Imaging 
System, Bruker BioSpin International AG, Switzerland).

Extra-Magnetic Field Mediated Tumor Targeting. IR-820 was loaded into the MMGNCs by adsorption. The balb/C mice were $6 \sim 8$ weeks old. By subcutaneously injection of $4 \mathrm{~T} 1$ cells suspension, the breast cancer model was established. In the $10^{\text {th }}$ day after the cells injection, IR-820 loaded MMGNCs were injected into the mouse by tail vessel injection. The mice were divided into two groups, each tumor of one group was attached with a magnet $(0.2 \mathrm{~T})$, and the other group obtained no further treatment. After 4 hour, all the mice were imaged by live fluorescence imaging (Xenogen IVIS spectrum, Caliper, PerkinElmer, USA, excitation wavelength was $745 \mathrm{~nm}$, emission wavelength was $840 \mathrm{~nm}$ ).

Infrared Thermal Imaging: in vitro and in vivo. The in vitro and in vivo evaluation of the photothermal performance of MMGNCs was measured by infrared thermal imaging (Fluke Ti32, USA).

\section{in vivo Therapy of $4 \mathrm{TI}$ cancer bearing Balb/C mice}

All the animal experiment procedures were carried out according to the standard procedures of State key laboratory of biotherapy, Sichuan University, China. Balb/C mice were supplied by Beijing HFK Bioscience Co., Ltd, China (female, 4 6weeks old). Breast cancer model was established by subcutaneous injection of $10^{6} 4 \mathrm{~T} 1$ cells per mouse.

Comparing of different drugs. Two anticancer model drugs, DOX and PTX, were chosen in therapy of $4 \mathrm{~T} 1$ cancer bearing Balb/C mice in vivo. Same dosages were administrated. While the diameter of the tumors grew to $\sim 6 \mathrm{~mm}, 32$ number of tumor bearing Balb/C mice were divided randomly into 4 groups. 4 groups of tumor bearing Balb/C female mice were treated by veil injection with normal saline (NS), blank MMGNCs, MMGNCs+DOX, MMGNCs+PTX, separately. Extra-MF mediated targeting (Before injection, magnets with the diameter of $\sim 6 \mathrm{~mm}$ were attached to the tumor site of the mice) and NIR irradiation (optical fiber coupled $808 \mathrm{~nm}$ high power laser diode (Hi-Tech Optoelec-tronics Co., Ltd. Beijing, China)) were applied in all groups. The dosage was settled at $2.5 \mathrm{~m} \mathrm{~g} / \mathrm{kg}$ mouse weight per administration. Tumor volume and body weight were measured in every two days interval.

Anticancer performance of DOX-loaded MMGNCs in vivo. While the diameter of the tumors grew to $\sim 6 \mathrm{~mm}$, the tumor bearing Balb/C mice were divided randomly into 10 groups. 10 groups of tumor bearing Balb/C female mice were treated by veil injection with Normal Saline, NS + laser, MMGNCs + laser, MMGNC + MF + laser, MMGNCs + DOX,
MMGNCs + DOX + MF, MMGNCs + DOX + laser, MMGNCs + DOX + MF + laser, free DOX with single administration, and free DOX with four administrations, separately. An optical fiber coupled $808 \mathrm{~nm}$ high power laser diode (Hi-Tech Optoelec-tronics Co., Ltd. Beijing, China) was used to irradiate tumors during our experiments. After the mice were intravenously injected with MMGNCs or DOX-loaded MMGNCs (DOX dosage was $2.5 \mathrm{mg} / \mathrm{kg}$ mice, DLC was $10.2 \%$ ) under extra-MF targeting to tumor for $4 \mathrm{~h}$, the tumor on each mouse was radiated by the $808 \mathrm{~nm}$ NIR laser at the power density of $2.5 \mathrm{~W} / \mathrm{cm}^{2}$ for $5 \mathrm{~min}$. The tumor sizes were measured by a caliper every the other day and calculated as the volume $=$ (tumor length) $\times(\text { tumor width })^{2} / 2$. For tremendous studies about the anticancer performance of free DOX have been reported, and it is well known that more free DOX applied, faster the mice died, due to the serious heart cytotoxicity of DOX, so in here, the data of the free DOX treated groups were not listed.

Histopathological studying. After administration, the organs and the tumors were harvested in predetermined timepoints, fixed in $10 \%$ neutral buffered formalin, processed routinely into paraffin, sectioned at $8 \mathrm{~mm}$, stained with hematoxylin \& eosin (H\&E), and examined by light microscopy. Examined tissues included: liver, spleen, lung, kidney, heart, and tumor.

Statistical analysis. Mean values and standard deviation were calculated for the amount of the drug release from MMGNCs as well as the results of tumor volume, IC50, body weight and Au and Fe concentration in tumor etc. Differences were considered significant at $p$ values $<0.05$. Statistical analysis was carried out by using SPSS 11.0 software (SPSS Inc., Chicago, IL, USA).

\section{Supplementary Material}

Table S1, Fig.S1 and S2.

http://www.thno.org/v04p0678s1.pdf

\section{Acknowledgements}

This work was financially supported by National Natural Science Foundation (NSFC31271021 and NSFC31222023), National Science and Technology Major Project (2011ZX09102-001-10), the International Science \& Technology Cooperation Program of China (2013DFG52300) and Chinese Key Basic Research Program (2010CB529906).

\section{Competing Interests}

The authors have declared that no competing interest exists. 


\section{References}

1. Schroeder A, Heller DA, Winslow MM, Dahlman JE, Pratt GW, Langer R, et al. Treating metastatic cancer with nanotechnology. Nat Rev Cancer. 2012; 12: 39-50.

2. Peer D, Karp JM, Hong S, Farokhzad OC, Margalit R, Langer R. Nanocarriers as an emerging platform for cancer therapy. Nat nanotechnol. 2007; 2: 751-60

3. Kim Y, Lee S, Kim Y, Kim B, Kang S. Multi-institutional phase I clinical trial of cremophor-free, polymeric micelle formulation of paclitaxel (Genexol-PM) with carboplatin for patients advanced epithelial ovarian cancer. Gynecol Oncol. 2012; 125: S32-S33.

4. Werner M, Cummings N, Sethi M, Wang E, Sukumar R, Moore D, et al. Preclinical evaluation of Genexol-PM, a nanoparticle formulation of paclitaxel, as a novel radiosensitizer for the treatment of non-small cell lung cancer. Inter J Radiat Oncol Boil Phys. 2013; 86: 463-68.

5. Dean M, Fojo T, Bates S. Tumour stem cells and drug resistence. Nat Rev Cancer. 2005; 5: 275-84;

6. Samson DJ, Seidenfeld J, Ziegler K, Aronson N. Chemotherapy sensitivity and resistance assays: A systematic review. J Clin Oncol. 2004; 22: 3618-30.

7. Bock C, Lengauer T. Managing drug resistance in cancer: lessons from HIV therapy. Nat Rev Cancer. 2012; 12: 494-501;

8. Bouwman P, Jonkers J. The effects of deregulated DNA damage signaling on cancer chemotherapy response and resistance. Nat Rev Cancer. 2012; 12: 587-98;

9. Higgins CF. Multiple molecular mechanisms for multidrug resistance transporters. Nature. 2007; 446: 749-57.

10. Li Z, Wang J, Fan S, Wu M, Li X, Li G. Nanoparticle delivery of anti-metastatic NM23-H1 gene improves chemotherapy in a mouse tumor model. Cancer Gene Ther. 2009; 16: 423-29.

11. Sas-Korczynska B, Sokollowski A, Korzeniowski S. The influence of time of raido-chemotherapy and other therapeutic factors on treatment results in patients with limited disease small cell lung cancer. Lung Cancer. 2013; 79: 14-19.

12. Chu C, Chen P, Bai L, Muo C, Song F, Chen S. Young nasopharyngeal cancer patients with radiotherapy and chemotherapy are most prone to ischaemic risk of stroke: a national database, controlled cohort study. Clin Otolaryngol. 2013; 38: 39-47.

13. Lee J, Chen K, Noh S, Garcia MA, Wang H, Lin W, et al. On-demand drug release system for in vivo cancer treatment through self-assembled magnetic nanoparticles. Angew Chem Int Ed. 2013; 52:4384-88.

14. Shao J, Griffin RJ, Galanzha EI, Kim J, Koonce N, Webber J, et al. Photothermal nanodrugs: potential of TNF-gold nanospheres for cancer theranostics. Sci Rep. 2013; 3: 1293-300.

15. Yang H, Hua M, Hwang T, Lin $K$, Huang $C$, Tsai R, et al. Non-invasive synergistic treatment of brain tumors by targeted chemotherapeutic delivery and amplified focused ultrasound-hyperthermia using magnetic nanographene oxide. Adv Mater. 2013; 25: 3605-11.

16. Skrabalak SE, Au L, Li X, Xia Y. Facile synthesis of Ag nanocubes and Au nancages. Nat Protoc. 2007; 2: 2182-90;

17. Kim JW, Galanzha EI, Shashkov EV, Moon HM, Zharov VP. Golden carbon nanotubes as multimodal photoacoustic and photothermal high-contrast molecular agnets. Nat Nanotechnol. 2009; 4: 688-94;

18. Rossella F, Soldano C, Bellani V, Tommasini M. Metal-filled carbon nanotubes as a novel class of photothermal nanomaterials. Adv Mater. 2012; 24: 2453-58;

19. Wang XJ, Wang C, Cheng L, Lee ST, Liu Z. noble metal coated single-walled carbon nanotubes for applications in surface enhanced Raman scattering imaging and photothermal therapy. J Am Chem Soc. 2012; 134: 7414-22.

20. Huang $X$, Tang $S, M u$ X, Dai $Y$, Chen $G$, Zhou Z, et al. Freestanding palladium nanosheets with plasmonic and catalytic properties. Nat Nanotechnol. 2011; 6: 28-32;

21. Jin MS, He GN, Zhang H, Zeng J, Xie ZX, Xia Y. Shape-controlled synthesis of copper nanocrystals in an aqueous solution with glucose as a reducing agent and hexadecylamine as a capping agent. Angew Chem Int Ed. 2011; 50: 10560-64;

22. Rycenga $\mathrm{M}$, Cobley $\mathrm{CM}$, Zeng J, Li W, Moran $\mathrm{CH}$, Zhang $\mathrm{O}$, et al. Controlling the synthesis and assembly of silver nanostructures for plasmonic applications. Chem Rev. 2011; 111: 3669-712.

23. Chaudhuri RG, Paria S. Core/shell nanoparticles: classes, properties, synthesis mechanisms, characterization, and applications. Chem Rev. 2012; 112: 2373-433;

24. Lim B, Xia Y. Metal nanocrystals with highly branched morphologies. Angew Chem Int Ed. 2011; 50: 76-85;

25. Zhang Z, Wang J, Chen C. Near-infrared light-mediated nanoplatforms for cancer thermo-chemotherapy and optical imaging. Adv Mater. 2013; 25: 3869-80.

26 Kang B, Mackey MA, El-Sayed MA. Nuclear targeting of gold nanoparticles in cancer cells induces DNA damage, causing cytokinesis arrest and apoptosis. J Am Chem Soc. 2010; 132: 1517-19.

27. Wang $Y$, Brown P, Xia Y. Nanomedicine: swarming towards the target. Nat Mater. 2011; 10: 482-83

28. Vanneman M, Dranoff G. Combining immunotherapy and targeted therapies in cancer treatment. Nat Rev Cancer. 2012; 12: 237-51.

29. Cheng L, Yang K, Li Y, Zeng X, Shao M, Lee ST, Liu Z. Multifunctional nanoparticles for upconversion luminescence/MR multimodal imaging and magnetically targeted photothermal therapy. Biomaterials. 2012;33: 2215-22;
30. Jang ES, Shin JH, Ren G, Park MJ, Cheng K, Chen X, et al. The manipulation of natural killer cells to target tumor sites using magnetic nanoparticles. Biomaterials. 2012; 33: 5584-92.

31. Mikhaylov G, Mikac U, Magaeva AA, Itin VI, Naiden EP, Psakhye I, et al. Ferri-liposomes as an MRI-visible drug-delivery system for targeting tumours and their microenvironment. Nat Nanotechnol. 2011; 6: 594-602.

32. Joyce JA, Pollard JW. Microenvironmental regulation of metastasis. Nat Rev Cancer. 2009; 9: 239-52

33. Psaila B, Lyden D. The metastatic niche: adapting the foreign soil. Nat Rev Cancer. 2009; 9: 285-93.

34. Reddy L, Arias J, Nicolas J, Couvreur P. magnetic nanoparticles: design and characterization, toxicity and biocompatibility, pharmaceutical and biomedical application. Chem Rev. 2012; 112: 5818-78.

35. Gao J, Gu H, Xu B. multifunctional magnetic nanoparticles: design, synthesis, and biomedical applications. Acc Chem Res. 2009; 42: 1097-107.

36. Chen $\mathrm{X}$, Tong $\mathrm{M}$. Solvothermal in-situ metal/ligand reactions: a new bridge between coordination chemistry and organic synthetic chemistry. Acc Chem Res. 2007; 40: 162-70.

37. Li H, Bian Z, Zhu J, Zhang D, Li G, Huo Y, et al. Mesoporous titania spheres with tunable chamber structure and enhanced photocatalytic activity. J Am Chem Soc. 2007: 129: 8406-07.

38. Yu S, Yoshimura M. Shape and phase control of ZnS nanocrystals: template fabrication of wurtzite $\mathrm{ZnS}$ single-crystal nanosheets and $\mathrm{ZnO}$ flake-like dendrites from a lamellar molecular precursor $\mathrm{ZnS}$. $\left(\mathrm{NH}_{2} \mathrm{CH}_{2} \mathrm{CH}_{2} \mathrm{NH}_{2}\right)_{0.5}$. Adv Mater. 2002; 14: 296-300

39. Farha O, Hupp J. Rational design, synthesis, purification, and activation of metal-organic framework materials. Acc Chem Res. 2010; 43: 1166-75.

40. Zacher D, Shekhah O, Woll C, Fischer R. Thin films of metal-organic frameworks. Chem Soc Rev. 2009;: 1418-29.

41. Yu H, Chen M, Rice PM, Wang SX, White RL, Sun S. dumbbell-like bifunctional Au-Fe3O4 nanoparticles. NANO Lett. 2005; 5: 379-82;

42. Xu C, Wang B, Sun S. Dumbbell-like Au-Fe3O4 nanoparticles for target-specific platin delivery. J Am Chem Soc. 2009; 131: 4216-17.

43. You J, Zhang G, Li C. Exceptionally high payload of doxorubicin in hollow gold nanospheres for near-infrared light-triggered drug release. ACS nano 2010; 4: 1033-41.

44 Tian J, Stella VJ. Degradation of paclitaxel and related compounds in aqueous solution II: nonepimerization degradation under neutral to basic $\mathrm{pH}$ conditions. J Pharm Sci. 2008; 97: 3100-08.

45. Gou $M$, Zheng $X$, Men $K$, Zhang J, Zheng $L$, Wang $X$, et al.

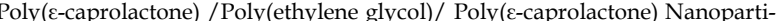
cles: Preparation, Characterization, and Application in Doxorubicin Delivery. J Phys Chem. B 2009; 113:12928-33.

46. Duff DG, Baiker A, Edwards PP. A new hydrosol of gold clusters. 1. Formation and particle size variation. Langmuir. 1993; 9: 2301-09. 